\title{
Calculation of the Dielectric Constant as a Function of Temperature Close to the Smectic A-Smectic B Transition in B5 Using the Mean Field Model
}

\author{
Hamit Yurtseven ${ }^{1}$ and Emel Kilit ${ }^{2}$ \\ ${ }^{1}$ Department of Physics, Middle East Technical University, 06531 Ankara, Turkey \\ ${ }^{2}$ Department of Physics, Yuzuncu Yil University, 65080 Van, Turkey \\ Correspondence should be addressed to Hamit Yurtseven, hamit@metu.edu.tr
}

Received 4 July 2012; Revised 24 September 2012; Accepted 25 September 2012

Academic Editor: Durga Ojha

Copyright (C) 2012 H. Yurtseven and E. Kilit. This is an open access article distributed under the Creative Commons Attribution License, which permits unrestricted use, distribution, and reproduction in any medium, provided the original work is properly cited.

The temperature dependence of the static dielectric constant $(\varepsilon)$ is calculated close to the smectic A-smectic $B\left(S_{A}-S_{B}\right)$ transition $\left(T_{c}=71.3^{\circ} \mathrm{C}\right)$ for the liquid crystal compound B5. By expanding the free energy in terms of the order parameter in the mean field theory, the expression for the dielectric susceptibility (dielectric constant) is derived and is fitted to the experimental data for $\varepsilon$ which was obtained at the field strengths of 0 and $67 \mathrm{kV} / \mathrm{cm}$ from literature. Coefficients in the free energy expansion are determined from our fit for the $S_{A}-S_{B}$ transition of B5. Our results show that the observed behaviour of the dielectric constant $\varepsilon$ close to the $S_{A}-S_{B}$ transition in $B 5$ can be described satisfactorily by our mean field model.

\section{Introduction}

Various smectic phases which occur in ferroelectric liquid crystals are of interest to study close to the phase transitions. In the smectic A phase, the long axes of the liquid crystal molecules are parallel to the director which is perpendicular to the smectic layers. In the smectic $\mathrm{C}$ (or $\mathrm{C}^{*}$ ) phase, those molecules are tilted (a tilt angle $\theta$ between the long axis and the director) and in the presence of the chiral molecules, the $\mathrm{AC}\left(\right.$ or $\mathrm{AC}^{*}$ ) transition becomes more interesting to study in ferroelectric liquid crystals. As in the smectic $\mathrm{C}$ (or $\mathrm{C}^{*}$ ) phase, the molecules are tilted in the smectic $\mathrm{G}$ phase and it has been observed experimentally [1] that the transitions between the ferroelectric phases (smectic AC, smectic A-G and smectic C-G) are influenced by an applied electric field. However, it has also been observed [1] that transitions between the nonferroelectric phases (smectic A-B, smectic B-E) are not influenced by an applied electric field. This has been demonstrated experimentally [1] for the nonferroelectric phases of smectic A, B, and E of compound $\mathrm{B} 5$ by measuring the temperature dependence of the dielectric constant at fixed field strengths.
Some theoretical models have been given in the literature to explain the transitions between the smectic phases. The mean field models where the free energy is expanded in terms of the order parameters with the coupling terms, have been used to analyze the experimental data. Regarding the spontaneous polarization $P$ and the tilt angle $\theta$, a bilinear coupling $(P \theta)[1,2]$ and biquadratic coupling $\left(P^{2} \theta^{2}\right)[3-5]$ in the free energy expansion have been used in the mean field models for the smectic $\mathrm{AC}$ (or $\mathrm{AC}^{*}$ ) transitions. In our earlier studies [6-10], we have also studied the mean field models with the $P^{2} \theta^{2}$ and $P \theta$ couplings for the $\mathrm{AC}$ (or $\mathrm{AC}^{*}$ ) transitions in ferroelectric liquid crystals.

In this study, we focus on the smectic A-B transition in compound B5 and we analyze the experimental data [1] for the temperature dependence of the dielectric constant $\varepsilon$ for constant electric fields of 0 and $67 \mathrm{kV} / \mathrm{cm}$. For this analysis, we use our mean field model [6] with the biquadratic coupling between the order parameters (polarization $P$ ) and a long-range bond-orientational order [11] for the smectic A-smectic B transition in B5.

Below, in Section 2 we give our mean field model for the smectic A-B transition. In Section 3, our calculations and 
results are given. We discuss our results in Section 4 and finally, conclusions are given in Section 5.

\section{Theory}

The smectic A-smectic B transition can be described by the free energy expanded in terms of the two-order parameters, namely, polarization $P$ (smectic A and B phases) and a longrange bond-orientational order $\psi$ (smectic B phase only) under an external electric field. Thus, the free energy can be written as

$$
g=\frac{1}{2} \alpha \psi^{2}+\frac{1}{4} b \psi^{4}+\frac{1}{6} c \psi^{6}+\frac{1}{2 \chi_{o} \varepsilon_{o}} P^{2}-D P^{2} \psi^{2}+\frac{1}{4} e P^{4} .
$$

Here, $\alpha=a\left(T-T_{c}\right)$ where $T_{c}$ is the transition temperature between the smectic $A$ and $B$ phases, $\chi_{0}$ is a constant dielectric susceptibility, and $\varepsilon_{0}$ is the permittivity. $a, b, c$, and e are constants and $D$ is the coupling constant in (1).

The temperature dependence of the polarization $P$ and the bond-orientational order $\psi$ can be obtained from the minimization of the free energy (1) with respect to $P$ and $\psi$, which gives

$$
\begin{gathered}
\alpha+b \psi^{2}+c \psi^{4}-2 D P^{2}=0, \\
\frac{1}{\chi_{o} \varepsilon_{o}}-2 D \psi^{2}+e P^{2}=0,
\end{gathered}
$$

respectively. Substituting (3) for $P^{2}$ into (2) then gives the free energy $g$ in terms of the bond-orientational order parameter $\psi$ only,

$$
g=\frac{1}{2} \alpha^{\prime} \psi^{2}+\frac{1}{4} b^{\prime} \psi^{4}+\frac{1}{6} c^{\prime} \psi^{6}
$$

with the new variables

$$
\alpha^{\prime}=\alpha+\frac{2 D}{e \chi_{0} \varepsilon_{0}}, \quad b^{\prime}=b-\frac{4 D^{2}}{e}, \quad c^{\prime}=c .
$$

Since (4) gives the free energy of the smectic B phase (in terms of the bond-orientational order parameter in this phase), the dielectric susceptibility $\chi_{\psi}$ can be obtained as a function of temperature by taking the second derivative of $g$ with respect to the $\psi$, which gives

$$
\chi_{\psi}^{-1}=a\left(T-T_{c}\right)+\frac{2 D}{e \chi_{o} \varepsilon_{o}}+a_{1} \psi^{2}+a_{2} \psi^{4},
$$

where $a_{1}=3 b^{\prime}(5)$ and $a_{2}=5 c$.

In order to predict the temperature dependence of the reciprocal dielectric susceptibility $\chi_{\psi}^{-1}(6)$, a functional form of $\psi$ is needed, which can be adopted from the molecular field theory [12] as follows:

$$
\begin{aligned}
& \psi \approx 1-2 \exp \left(-\frac{2 T_{c}}{T}\right), \quad T \ll T_{c}, \\
& \psi \approx\left[3\left(1-\frac{T}{T_{c}}\right)\right]^{1 / 2}, \quad 0<T_{c}-T \ll T_{c}, \\
& \psi \approx 0, \quad T_{c}<T
\end{aligned}
$$

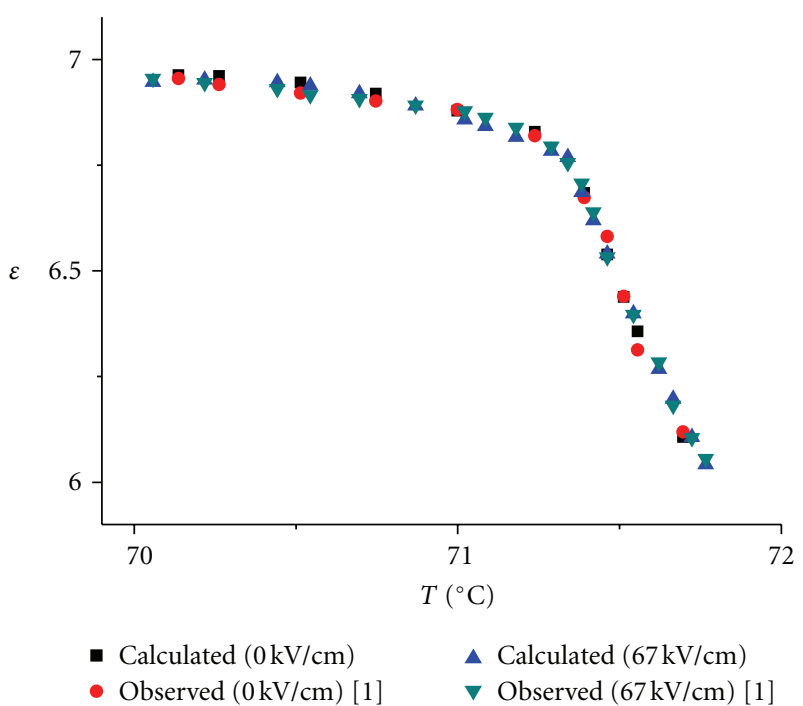

FIGURE 1: The dielectric constant $\varepsilon$ calculated from (6) as a function of temperature for the smectic A-smectic B transition under the dielectric fields of 0 and $67 \mathrm{kV} / \mathrm{cm}$ for compound B5. The observed data [1] is also shown here.

for the long-range bond-orientational order parameter in the smectic B phase. Close to the smectic A-smectic B transition, a power law formula is valid, as given in (7).

\section{Calculations and Results}

We analyzed the temperature dependence of the dielectric susceptibility according to (6) which was fitted to the experimental data for the dielectric constant $\varepsilon[1](\chi=\varepsilon-1)$ close to the smectic A-smectic B transition in B5. In (6), we first calculated the temperature dependence of the order parameter $\psi$ using the power-law formula (7) where the temperature for the smectic $\mathrm{A}$-smectic $\mathrm{B}$ transition was taken as $T_{c}=71.3^{\circ} \mathrm{C}$ at zero electric field $(E=0)$ in B5. By fitting (6) to the experimental $\varepsilon$ data [1], the fitted parameters $a$, $a_{1}$ and $a_{2}$ below $T_{c}$ were obtained $(E=0)$, as given in Table 1. Similarly, (6) was fitted to the experimental $\varepsilon$ data [1] for the electric field of $67 \mathrm{kV} / \mathrm{cm}$ close to the smectic A-smectic B transition with the fitted parameters (Table 1). Values of $2 D / e \chi_{o} \varepsilon_{o}$ were deduced from the experimental $\varepsilon$ data [1] when $\psi=0$ at $T=T_{c}$ (6). Since the transition temperatures are not induced by the external electric field for nonferroelectric phases [1], we took the same transition temperature $\left(T_{c}=71.3^{\circ} \mathrm{C}\right)$ for $67 \mathrm{kV} / \mathrm{cm}$ in $\mathrm{B} 5$, as shown in Figure 1. Thus, we get the same fits (6) for the fixed bias field strengths of 0 and $67 \mathrm{kV} / \mathrm{cm}$ for the smectic Asmectic B transition in B5 (Figure 1). In the same manner, we analyzed the experimental data for the dielectric constant $\varepsilon$ [1] according to (1) with $\psi=0$ for $T>T_{c}$ using the fixed electric fields of 0 and $67 \mathrm{kV} / \mathrm{cm}$. Values of the fitted parameter a are given within the temperature intervals in Table 2. This data is also plotted in Figure 1 with the observed data [1]. 
TABLE 1: Values of the coefficients according to (6) for the smectic A-smectic B transition below $T_{c}$ within the temperature interval under the electric fields indicated for compound B5.

\begin{tabular}{lcccccc}
\hline$E(\mathrm{kV} / \mathrm{cm})$ & $T_{c}\left({ }^{\circ} \mathrm{C}\right)$ & $a\left({ }^{\circ} \mathrm{C}^{-1}\right)$ & $a_{1}$ & $a_{2}$ & $2 D / e \chi_{o} \varepsilon_{o}$ & Temperature interval $\left({ }^{\circ} \mathrm{C}\right)$ \\
\hline 0 & 71.3 & 0.056 & 1.16 & 1.70 & 0.172 & $70.14<T<71.24$ \\
67 & 71.3 & 0.057 & 1.13 & 2.53 & 0.173 & $70.06<T<71.29$ \\
\hline
\end{tabular}

TABLE 2: Values of the coefficients according to (6) for the smectic A-smectic B transition above $T_{c}$ within the temperature interval under the electric fields indicated for compound B5.

\begin{tabular}{lcccc}
\hline$E(\mathrm{kV} / \mathrm{cm})$ & $T_{c}\left({ }^{\circ} \mathrm{C}\right)$ & $a\left({ }^{\circ} \mathrm{C}^{-1}\right)$ & $2 D /$ e $_{o} \varepsilon_{o}$ & Temperature interval $\left({ }^{\circ} \mathrm{C}\right)$ \\
\hline 0 & 71.3 & 0.065 & 0.170 & $71.39<T<71.70$ \\
67 & 71.3 & 0.059 & 0.171 & $71.34<T<71.77$ \\
\hline
\end{tabular}

\section{Discussion}

We analyzed here the temperature dependence of the dielectric constant $\varepsilon$ through the reciprocal dielectric susceptibility $\chi^{-1}$ (6) in our mean field model with the biquadratic $\left(P^{2} \psi^{2}\right)$ coupling for the smectic A-smectic B transition in B5. As pointed out previously, transition temperatures are not shifted for the external electric fields of 0 and $67 \mathrm{kV} / \mathrm{cm}$ (Figure 1) due to the fact that the smectic A and smectic $\mathrm{B}$ phases are nonferroelectric of compound B5. For the ferroelectric transitions of smectic A-smectic C, smectic Asmectic $G$ and smectic C-smectic G of compound A6, it has been observed experimentally [1] that the transition temperatures are shifted under various fixed field strengths. It has also been observed [1] that nonferroelectric phases (smectic B-smectic E) are not influenced by an electric field for this compound B5 studied here. Regarding nonferroelectric phases, in fact the smectic A phase at high temperatures occurs at zero electric field only since the external electric field induces a tilt angle which exists in the smectic $C$ phase. The tilted smectic phase possesses a spontaneous electric polarization $P_{s}$ if the molecules have a permanent dipole moment and they are chiral [13].

As shown in Figure 1, dielectric constant $\varepsilon$ increases abruptly with the decreasing temperature near the smectic A-smectic B transition $\left(T_{c}=71.3^{\circ} \mathrm{C}\right)$ of compound B5. This is due to the larger electroclinic effect in the smectic B phase $[1]$.

As we pointed out previously, the dielectric constant (or dielectric susceptibility) was calculated as a function of temperature (6) from our mean field model with the biquadratic $P^{2} \psi^{2}$ coupling (1) for the smectic A-smectic B transition of compound B5 (Figure 1). In this model, the long-range bond-orientational order parameter $\psi$ was considered as the primary order parameter and the polarization $P$ as the secondary order parameter in the free energy expansion (1). With the biquadratic coupling $\left(P^{2} \psi^{2}\right)$, quadrupolar interactions between the molecules are attributed to the mechanism of the smectic A-smectic B transition of compound B5 in our mean field model. Regarding the temperature dependence of the dielectric constant $\varepsilon$ calculated from (6) which was fitted to the experimental data [1] as stated above, our mean field model describes the observed behaviour of $\varepsilon$ satisfactorily for the smectic A-smectic B transition of compound B5.
This indicates that the main mechanism for this transition is due to quadrupole-quadrupole interactions which involve a long-range bond-orientational ordering in B5.

\section{Conclusions}

The dielectric constant $\varepsilon$ was predicted using our mean field model with the biquadratic coupling $P^{2} \psi^{2}(P$ is the polarization and the $\psi$ is the long-range bond-orientational order parameter) for the smectic A-smectic B transition for constant electric fields in compound B5. The predicted $\varepsilon$ was fitted to the experimental data from the literature and the fitted parameters were determined for the smectic Asmectic B transition of compound B5. It was shown here that our mean field model describes the observed behaviour of the dielectric constant adequately for this transition of the liquid crystalline material studied here. The observed data also shows that the transition temperatures are not shifted under the electric field for this nonferroelectric transition.

\section{References}

[1] Ch. Bahr, G. Heppke, and B. Sabaschus, "Influence of an electric field on phase transitions in ferroelectric liquid crystals," Liquid Crystals, vol. 11, no. 1, pp. 41-48, 1992.

[2] Ch. Bahr and G. Heppke, "Influence of electric field on a firstorder smectic-A-ferroelectric-smectic-C liquid-crystal phase transition: a field-induced critical point," Physical Review A, vol. 41, pp. 4335-4342, 1990.

[3] C. C. Huang and S. Dumrongrattana, "Generalized mean-field model for the smectic-A chiral-smectic C phase transition," Physical Review A, vol. 34, no. 6, pp. 5020-5026, 1986.

[4] T. Carlsson, B. Zeks, A. Levstik, C. Filipic, I. Levstik, and R. Blinc, "Generalized Landau model of ferroelectric liquid crystals," Physical Review A, vol. 36, no. 3, pp. 1484-1487, 1987.

[5] R. Blinc, "Models for phase transitions in ferroelectric liquid crystals: theory and experimental results," in Phase Transitions in Liquid Crystals, S. Martellucci and A. N. Chester, Eds., Plenum Press, New York, NY, USA, 1992.

[6] S. Salihoğlu, H. Yurtseven, A. Giz, D. Kayışoğlu, and A. Konu, "The mean field model with $P^{2} \theta^{2}$ coupling for the smectic A-smectic C* phase transition in liquid crystals," Phase Transitions, vol. 66, pp. 259-270, 1998. 
[7] S. Salihoğlu, H. Yurtseven, and B. Bumin, "Concentration dependence of polarization for the $\mathrm{AC}^{*}$ phase transition in a binary mixture of liquid crystals," International Journal of Modern Physics B, vol. 12, no. 20, pp. 2083-2090, 1998.

[8] H. Yurtseven and E. Kilit, "Temperature dependence of the polarization and tilt angle under an electric field close to the smectic AC* phase transition in a ferroelectric liquid crystal," Ferroelectrics, vol. 365, no. 1, pp. 122-129, 2008.

[9] E. Kilit and H. Yurtseven, "Calculation of the dielectric constant as a function of temperature near the smectic AC* phase transition in ferroelectric liquid crystals," Ferroelectrics, vol. 365, no. 1, pp. 130-138, 2008.

[10] H. Yurtseven and M. Kurt, "Tilt angle and the temperature shifts calculated as a function of concentration for the AC* phase transition in a binary mixture of liquid crystals," International Journal of Modern Physics B, vol. 25, no. 13, pp. 1791-1806, 2011.

[11] Z. Kutnjak and C. W. Garland, "Generalized smectic-hexatic phase diagram," Physical Review E, vol. 57, no. 3, pp. 30153020, 1998.

[12] R. Brout, Phase Transitions, chapter 2, Benjamin, New York, NY, USA, 1965.

[13] R. B. Meyer, L. Liebert, L. Strzelecki, and P. Keller, "Ferroelectric liquid crystals," Journal de Physique Lettres, vol. 36, no. 3, pp. 69-71, 1975. 

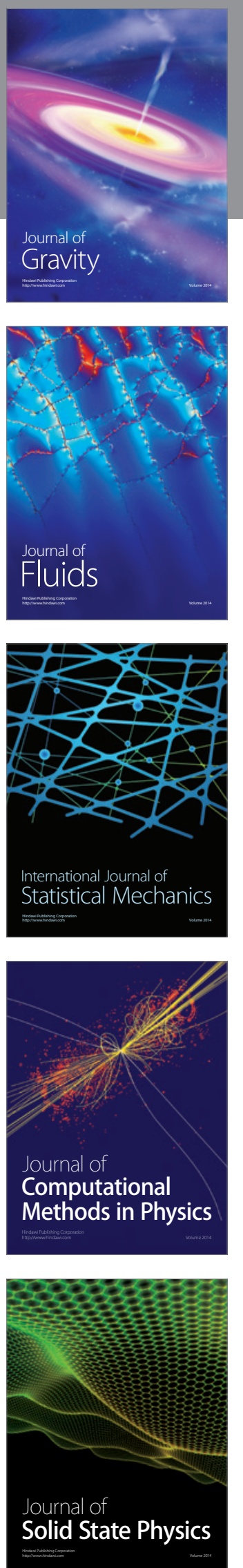

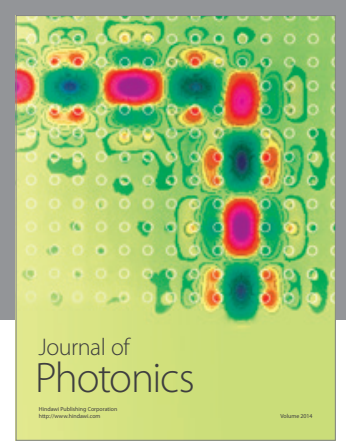

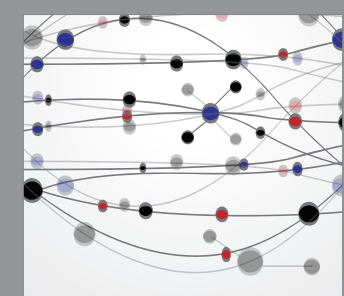

The Scientific World Journal
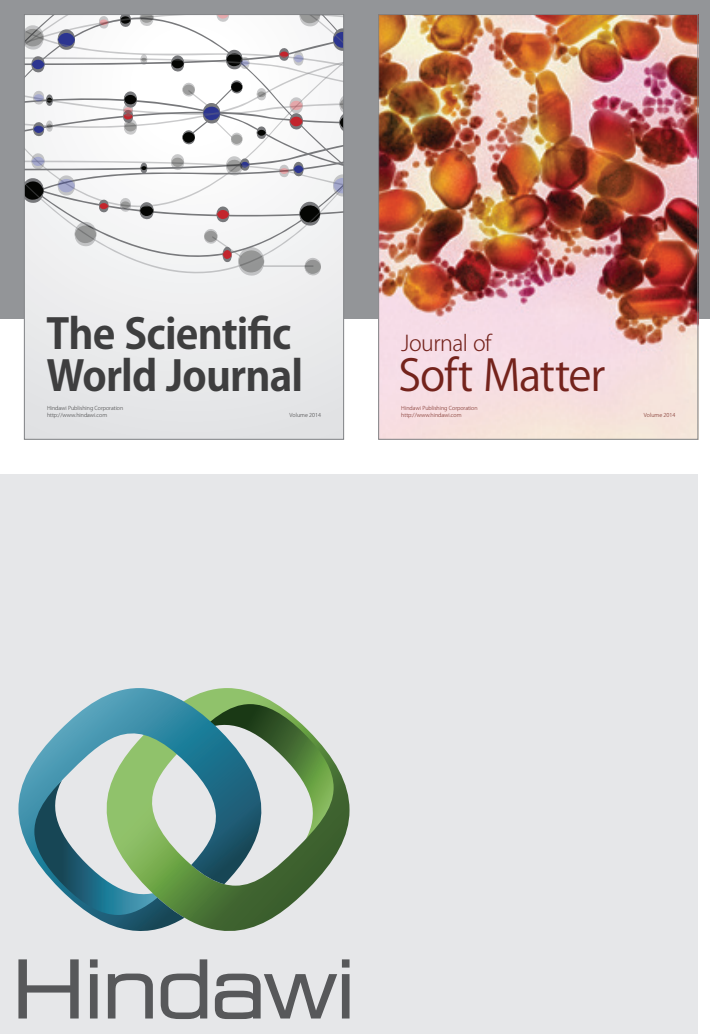

Submit your manuscripts at

http://www.hindawi.com
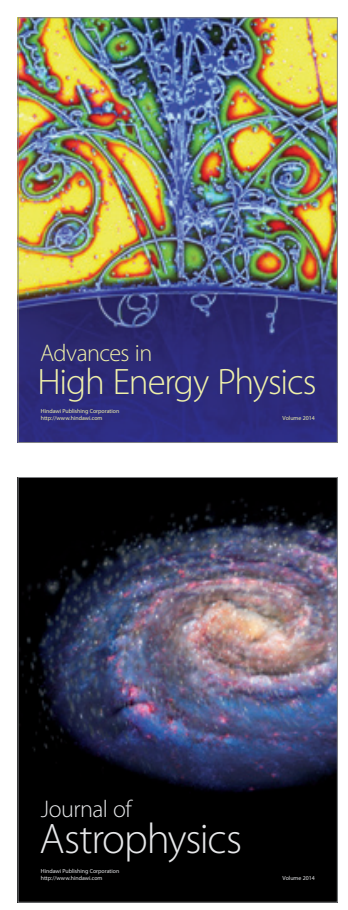
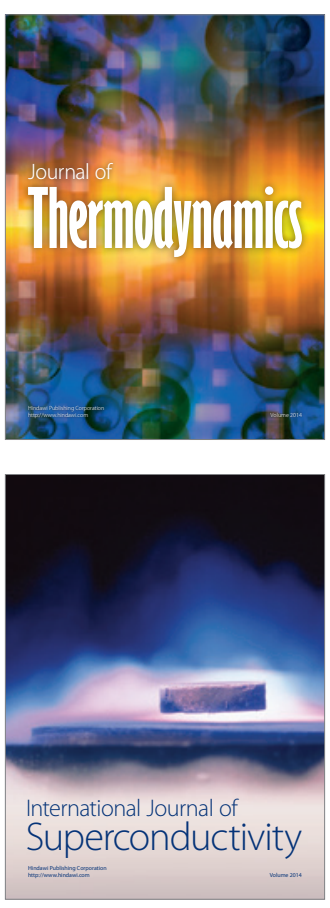
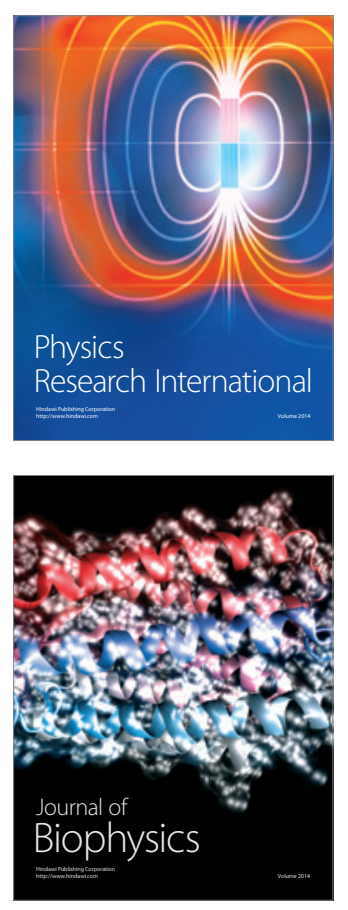
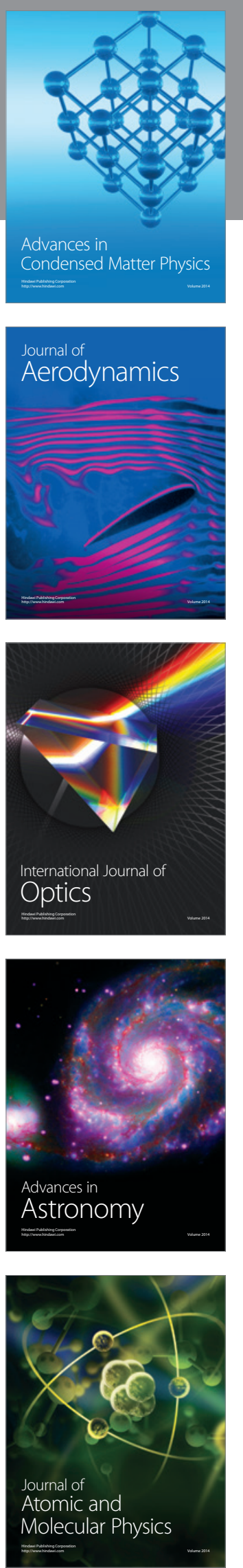